As part of the 50th birthday celebration, we are arranging to reprint 12 papers, the Editor's Choice, which have appeared in previous issues of the fournal. They have been chosen partly to illustrate the range and scope of the fournal over the years and partly because they are or were important in their day. More significantly, they have been chosen because they exemplify some of the best in scientific writing and can all be read with great pleasure and all who wish to communicate their observations, their ideas, or their enthusiasms would do well to study them and learn from them.

We will publish one paper each month through the year and they will appear in the order in which they were originally published.

Editor's Choice

\title{
Dust exposure and mortality in chrysotile mining, 1910-75
}

by J C McDonald, F D K Liddell, G W Gibbs, G E Eyssen, and A D McDonald

(British fournal of Industrial Medicine 1980;37:11-24)

Corbett McDonald has had a distinguished and varied career at the Public Health Laboratory Service, at McGill University and at the London School of Hygiene and Tropical Medicine, and now-in so called retirement-at the National Heart and Lung Institute. His outstanding contribution to the field of occupational epidemiology has been in the field of asbestos related diseases. The present paper describes almost the end result of a study begun in 1966 and carried on for 12 years on a cohort of 11379 workers exposed to chrysotile in the asbestos mines and mills of Quebec. This work elegantly demonstrates that "Essentially linear relationships [exist] between indices of exposure, based on dust concentration (mpcf) multiplied by length of service, and lung. cancer, pneumoconiosis, and total number of deaths". Furthermore, McDonald and his col- leagues go on to say that they "are now able to assess, with reasonable confidence, the mortality risk associated with work at different levels of dust concentrations". They also claimed that it would be possible, "for social decisions to be made as to what level, if any, is 'acceptable'."

McDonald's work over the years showed conclusively that the development of asbestos related diseases was a function of the degree and duration of exposure and laid low the fallacy of the notion that a single fibre could cause cancer. One might have expected his observations would have been welcomed, implying as they did that there were conditions under which asbestos could be worked without harm to health. But this was not to be so. $\mathrm{He}$ and his colleagues were subjected to a campaign of vituperation that was designed to achieve political rather than scientific ends. So far as I know he has never fully given an account of this episode and he may find it too painful to do so, although it would certainly be enlightening and instructive. 